\title{
Groups of virtual trefoil and Kishino knots
}

\author{
V. G. Bardakov, Yu. A. Mikhalchishina, M. V. Neshchadim
}

April 18, 2018

\begin{abstract}
In the paper [13] for an arbitrary virtual link $L$ three groups $G_{1, r}(L), r>0, G_{2}(L)$ and $G_{3}(L)$ were defined. In the present paper these groups for the virtual trefoil are investigated. The structure of these groups are found out and the fact that some of them are not isomorphic to each other is proved. Also we prove that $G_{3}$ distinguishes the Kishino knot from the trivial knot. The fact that these groups have the lower central series which does not stabilize on the second term is noted. Hence we have a possibility to study these groups using quotients by terms of the lower central series and to construct representations of these groups in rings of formal power series. It allows to construct an invariants for virtual knots.
\end{abstract}

\section{Introduction}

Unlike classical knots virtual ones are not topological objects therefore there is no natural definition of a virtual knot group. There exist several approaches to the definition (see [11, 16, 1, 9, 2, 8, 4, 15, 13]). Some of the approaches use a knot diagram, other ones use representations of the virtual braid group by automorphisms of some groups. The following natural problems occur of how to compare these groups, how to describe isomorphic groups and how to construct homomorphisms of some groups into another ones. A basis of such an analysis was put in [5].

In the present work groups defined by Yu. A. Mikhalchishina [13] are analyzed. In [13] representations $W_{1, r}, r>0, W_{2}$ and $W_{3}$ of the virtual braid group $V B_{n}$ into the automorphism $\operatorname{group} \operatorname{Aut}\left(F_{n+1}\right)$ of a free group $F_{n+1}=\left\langle y, x_{1}, x_{2}, \ldots, x_{n}\right\rangle$ of rank $n+1$ were constructed. These representations extend Wada representations [17] $w_{1, r}, r>0, w_{2}, w_{3}$ of the braid group $B_{n}$ into the automorphism group $\operatorname{Aut}\left(F_{n}\right)$ of a free group $F_{n}=\left\langle x_{1}, x_{2}, \ldots, x_{n}\right\rangle$ of rank $n$. Using these representations, for each virtual link $L$ the groups $G_{1, r}(L), G_{2}(L)$ and $G_{3}(L)$ were defined. In particular, generators and defining relations of the groups for the virtual trefoil were written. 
In the present work the fact that groups of the virtual trefoil $T_{v} G_{1, r}\left(T_{v}\right)$ and $G_{2}\left(T_{v}\right)$ are not isomorphic to each other will be proven.

Note that the most of virtual knot groups do not distinguish the group of the Kishino knot from the trivial one. The group defined in [9] is an exception. In the paper [13] the fact that groups constructed using representations $W_{1, r}$ and $W_{2}$ do not distinguish the Kishino knot from the trivial one is stated. And the natural question whether the group constructed using the representation $W_{3}$ distinguishes the Kishino knot from the trivial one was formulated in [13]. In present work the positive answer on the question is given.

As it is well known if $G$ is a group of a classical knot, then its commutator subgroup coincides with the third term of lower central series, i. e. $[G, G]=[[G, G], G]$. Therefore, the factorization by terms of the lower central series cannot be used for distinguish of knots. On the other hand as it was pointed out in the paper [3] some of virtual link groups are residually nilpotent groups. Recall that a group $G$ is referred to as residually nilpotent if for every nontrivial element $g$ from $G$ there exists a homomorphism $\varphi: G \longrightarrow N$ on a nilpotent group $N$ such that $\varphi(g) \neq 1$. It is easy to observe that $G$ is residually nilpotent if and only if the intersection of all terms of its lower central series is a trivial group.

In the paper [6] was noted that the virtual trefoil group from [1] has different first five terms of lower central series. This fact allows to construct new invariants of virtual links. The question occurs whether virtual link groups are residually nilpotent or not. Moreover in [6] representations of some virtual knot groups into finite algebras were constructed. In the present paper this approach is being developed.

Let us describe the paper content. In Section 2 we recall representations constructed in [13] of the virtual braid group $V B_{n}$ into the group of automorphisms of free groups which are extensions of the Wada representations of braid group $B_{n}$. Also we give presentations of three groups $G_{1, r}, G_{2}$ and $G_{3}$ of the virtual trefoil. In Section 3 the structure of these groups is found out. In Section 4, using quotients by the second commutator subgroups, we prove that $G_{1, r}$ is not isomorphic to $G_{2}$; we study the quotients by the terms of the lower central series and prove that $\gamma_{2}\left(G_{2}\right) / \gamma_{3}\left(G_{2}\right)$ is the cyclic group of order 2 and the quotient $\gamma_{k}\left(G_{2}\right) / \gamma_{k+1}\left(G_{2}\right)$ has exponent 4 for all $k \geq 2$. Studying the lower central series for $G_{1, r}$, we prove that $G_{1, r} / \gamma_{4}\left(G_{1, r}\right) \cong F_{2} / \gamma_{4}\left(F_{2}\right)$ and $\gamma_{4}\left(G_{1, r}\right) / \gamma_{5}\left(G_{1, r}\right) \cong \mathbb{Z} \times \mathbb{Z} \times \mathbb{Z}_{r}$. In Section 5 we prove that $G_{3}(K i)$ of the Kishino knot $K i$ is not isomorphic to $G_{3}(U)$ of the trivial knot $U$. In Section 6 we construct a representation of an arbitrary finitely presented group into a ring of formal power series with non-commutative variables and prove that the image does not depend on the presentation of group; we construct representations of $G_{1, r}$ and $G_{2}$ into some finite dimensional algebra.

Acknowledgement. This work was supported by the Russian Science Foundation (project No. 16-41-02006). The authors are also grateful to E. I. Timoshenko for the consultation on metabelian groups. 


\section{Extensions of Wada representations and virtual trefoil groups}

In the paper [13] the following three representations of the virtual braid group $V B_{n}$ into the automorphism group $\operatorname{Aut}\left(F_{n+1}\right)$ of the rank $n+1$ free group $F_{n+1}=\left\langle y, x_{1}, x_{2}, \ldots, x_{n}\right\rangle$ were constructed.

1. The representation $W_{1, r}, r>0$ is defined by the action on the generators

$$
W_{1, r}\left(\sigma_{i}\right):\left\{\begin{array}{l}
x_{i} \longmapsto x_{i}^{r} x_{i+1} x_{i}^{-r}, \\
x_{i+1} \longmapsto x_{i},
\end{array} \quad W_{1, r}\left(\rho_{i}\right):\left\{\begin{array}{l}
x_{i} \longmapsto x_{i+1}^{y^{-1}} \\
x_{i+1} \longmapsto x_{i}^{y}
\end{array}\right.\right.
$$

Here and onward we point out only nontrivial actions on generators assuming that other generators are fixed.

2. The representation $W_{2}$ is defined by the action on the generators

$$
W_{2}\left(\sigma_{i}\right):\left\{\begin{array}{l}
x_{i} \longmapsto x_{i} x_{i+1}^{-1} x_{i}, \\
x_{i+1} \longmapsto x_{i},
\end{array} \quad W_{2}\left(\rho_{i}\right):\left\{\begin{array}{l}
x_{i} \longmapsto x_{i+1}^{y^{-1}} \\
x_{i+1} \longmapsto x_{i}^{y}
\end{array},\right.\right.
$$

3. The representation $W_{3}$ is defined by the action on the generators

$$
W_{3}\left(\sigma_{i}\right):\left\{\begin{array}{l}
x_{i} \longmapsto x_{i}^{2} x_{i+1}, \\
x_{i+1} \longmapsto x_{i+1}^{-1} x_{i}^{-1} x_{i+1},
\end{array} \quad W_{3}\left(\rho_{i}\right):\left\{\begin{array}{l}
x_{i} \longmapsto x_{i+1}^{y^{-1}} \\
x_{i+1} \longmapsto x_{i}^{y}
\end{array},\right.\right.
$$

These representations extend Wada representations [17] $w_{1, r}, r>0, w_{2}, w_{3}$ of the braid group $B_{n}$ into the automorphisms group $\operatorname{Aut}\left(F_{n}\right)$ of the rank $n$ free group $F_{n}=\left\langle x_{1}, x_{2}, \ldots, x_{n}\right\rangle$.

In the paper [13] for each virtual link three types of groups were defined: $G_{1, r}(L), G_{2}(L)$ and $G_{3}(L)$ that correspond to described representations. And the fact that these groups are invariants of a virtual link $L$ is proved. In particular, for the virtual trefoil these groups have the form:

$$
\begin{aligned}
& G_{1, r}=\left\langle x, y \| x^{-r} y^{-1} x y x^{r}=y^{-2} x^{r} y x y^{-1} x^{-r} y^{2}\right\rangle, \quad r>0, \\
& G_{2}=\left\langle x, y \| x y^{-1} x^{-1} y x=y^{-2} x y x^{-1} y^{-1} x y^{2}\right\rangle, \\
& G_{3}=\left\langle x_{1}, x_{2}, y \| x_{1}=y x_{2}^{-1} x_{1}^{-1} x_{2}^{-1} x_{1}^{-1} x_{2} y^{-1}, x_{2}=y^{-1} x_{1}^{2} x_{2} x_{1} x_{2} y\right\rangle .
\end{aligned}
$$

Note that the group $G_{1,1}$ is isomorphic to the group found out in [2] (see also [4]).

\section{$3 \quad$ Structure of the virtual trefoil groups}

The structure of the virtual trefoil groups gives 
Theorem 3.1 1) The group $G_{1, r}$ is the semi-direct product $H 入 \mathbb{Z}$, where

$$
H=\ldots \underset{B_{-2}}{*} A_{-1} \underset{B_{-1}}{*} A_{0} \underset{B_{0}}{*} A_{1} \underset{B_{1}}{*} \ldots
$$

is the amalgamated free product with

$A_{i}=\left\langle x_{i-1}, x_{i}, x_{i+1} \|\left[x_{i}, x_{i-1}^{\varepsilon} x_{i+1}^{-\varepsilon}\right]=1\right\rangle \cong\left(\left\langle x_{i}\right\rangle \times\left\langle x_{i-1}^{\varepsilon} x_{i+1}^{-\varepsilon}\right\rangle\right) *\left\langle x_{i+1}\right\rangle=(\mathbb{Z} \times \mathbb{Z}) * \mathbb{Z}$ if $r=\varepsilon= \pm 1$,

and

$$
A_{i}=\left\langle x_{i-1}, x_{i}, x_{i+1} \| x_{i}^{-1}\left(x_{i-1}^{r} x_{i+1}^{-r}\right) x_{i}=x_{i-1}^{r} x_{i+1}^{-r}\right\rangle \text { if } r=\varepsilon= \pm 1 ;
$$

all $B_{i}$ are isomorphic to $F_{2}$.

2) $G_{2}$ is the semi-direct product $H 入 \mathbb{Z}$, where

$$
H=\ldots \underset{B_{-2}}{*} A_{-1} \underset{B_{-1}}{*} A_{0} \underset{B_{0}}{*} A_{1} \underset{B_{1}}{*} \ldots
$$

is the amalgamated free product with

$$
A_{i}=\left\langle x_{i-1}, x_{i}, x_{i+1} \|\left(x_{i-1} x_{i+1}^{-1}\right)^{x_{i} x_{i-1}^{-1}}=\left(x_{i-1} x_{i+1}^{-1}\right)^{-1}\right\rangle
$$

and all $B_{i}$ are isomorphic to $F_{2}$.

3) $G_{3}$ is an HNN-extension:

$$
G_{3}=\left\langle x_{1}, x_{2}, y \| y^{-1} A y=B, \varphi\right\rangle
$$

with the base group $\left\langle x_{1}, x_{2}\right\rangle=F_{2}$ and associated subgroups $A=\left\langle x_{2} x_{1} x_{2}, x_{1}\right\rangle, B=\left\langle x_{1} x_{2} x_{1}, x_{2}\right\rangle$, which are also isomorphic to $F_{2}$.

Proof. 1) Consider the group $G_{1, r}$. Let

$$
x_{i}=y^{-i} x y^{i}, \quad i \in \mathbb{Z}
$$

Then the defining relation of the group $G_{1, r}$ can be rewritten in the form

$$
x_{0}^{-r} x_{1} x_{0}^{r}=x_{2}^{-r} x_{1} x_{2}^{r} .
$$

Conjugating the relation by $y^{i}, i \in \mathbb{Z}$, we have

$$
x_{i}^{-r} x_{i+1} x_{i}^{r}=x_{i+2}^{-r} x_{i+1} x_{i+2}^{r}, \quad i \in \mathbb{Z} .
$$

Let us define the following groups

$$
\begin{gathered}
H=\left\langle x_{i}, i \in \mathbb{Z} \| x_{i}^{-r} x_{i+1} x_{i}^{r}=x_{i+2}^{-r} x_{i+1} x_{i+2}^{r}, i \in \mathbb{Z}\right\rangle, \\
A_{i}=\left\langle x_{i-1}, x_{i}, x_{i+1} \| x_{i-1}^{-r} x_{i} x_{i-1}^{r}=x_{i+1}^{-r} x_{i} x_{i+1}^{r}\right\rangle
\end{gathered}
$$




$$
B_{i}=\left\langle x_{i}, x_{i+1}\right\rangle
$$

Then the group $G_{1, r}$ can be decomposed into the semidirect product

$$
G_{1, r}=H 入 \mathbb{Z}
$$

where $\mathbb{Z}=\langle y\rangle$ is the infinite cyclic group generated by the element $y$, the group $H$ is the amalgamated free product

$$
H=\ldots \underset{B_{-2}}{*} A_{-1} \underset{B_{-1}}{*} A_{0} \underset{B_{0}}{*} A_{1} \underset{B_{1}}{*} \ldots
$$

Since $A_{i}$ is a group with one relation the subgroup $B_{i}$ is a free group of rank 2 .

The defining relation of the subgroup $A_{i}$ of the group $G_{1, r}$ can be rewritten in the form

$$
\left[x_{i}, x_{i-1}^{r} x_{i+1}^{-r}\right]=1 \text {. }
$$

If $r=\varepsilon= \pm 1$, then

$$
A_{i}=\left\langle x_{i-1}, x_{i}, x_{i+1} \|\left[x_{i}, x_{i-1}^{\varepsilon} x_{i+1}^{-\varepsilon}\right]=1\right\rangle \cong\left(\left\langle x_{i}\right\rangle \times\left\langle x_{i-1}^{\varepsilon} x_{i+1}^{-\varepsilon}\right\rangle\right) *\left\langle x_{i+1}\right\rangle=(\mathbb{Z} \times \mathbb{Z}) * \mathbb{Z}
$$

if $r \neq \pm 1$, then the group $A_{i}$ is the $H N N$-extension:

$$
A_{i}=\left\langle x_{i-1}, x_{i}, x_{i+1} \| x_{i}^{-1}\left(x_{i-1}^{r} x_{i+1}^{-r}\right) x_{i}=x_{i-1}^{r} x_{i+1}^{-r}\right\rangle .
$$

2) Consider the group $G_{2}$. Let

$$
x_{i}=y^{-i} x y^{i}, \quad i \in \mathbb{Z}
$$

Then the defining relation of the group $G_{2}$ can be rewritten in the form

$$
x_{0} x_{1}^{-1} x_{0}=x_{2} x_{1}^{-1} x_{2} .
$$

Conjugating this relation by $y^{i}, i \in \mathbb{Z}$, we obtain

$$
x_{i} x_{i+1}^{-1} x_{i}=x_{i+2} x_{i+1}^{-1} x_{i+2}, \quad i \in \mathbb{Z} .
$$

Define the following groups

$$
\begin{gathered}
H=\left\langle x_{i}, i \in \mathbb{Z} \| x_{i} x_{i+1}^{-1} x_{i}=x_{i+2} x_{i+1}^{-1} x_{i+2}, i \in \mathbb{Z}\right\rangle, \\
A_{i}=\left\langle x_{i-1}, x_{i}, x_{i+1} \| x_{i-1} x_{i}^{-1} x_{i-1}=x_{i+1} x_{i}^{-1} x_{i+1}\right\rangle, \\
B_{i}=\left\langle x_{i}, x_{i+1}\right\rangle .
\end{gathered}
$$

Then the group $G_{2}$ can be decomposed into the semidirect product

$$
G_{2}=H 入 \mathbb{Z}
$$


where $\mathbb{Z}=\langle y\rangle$ is the infinite cyclic group generated by the element $y$, the group $H$ is the amalgamated free product

$$
H=\ldots \underset{B_{-2}}{*} A_{-1} \underset{B_{-1}}{*} A_{0} \underset{B_{0}}{*} A_{1} \underset{B_{1}}{*} \ldots
$$

The defining relation of the subgroup $A_{i}$ of the group $G_{2}$ can be rewritten in the form

$$
x_{i}^{-1}\left(x_{i-1} x_{i+1}^{-1}\right) x_{i}=x_{i-1}^{-1} x_{i+1} \Leftrightarrow\left(x_{i-1} x_{i+1}^{-1}\right)^{x_{i} x_{i-1}^{-1}}=\left(x_{i-1} x_{i+1}^{-1}\right)^{-1} .
$$

Therefore, the group $A_{i}$ is the $H N N$-extension and it contains the subgroup which is isomorphic to the fundamental group of the Klein bottle.

Since $A_{i}$ is a group with one relation the subgroup $B_{i}$ is a free group of rank 2 .

3) Rewrite relations of the group $G_{3}$ in the form

$$
x_{1}^{y}=x_{2}^{-1} x_{1}^{-1} x_{2}^{-1} x_{1}^{-1} x_{2}, \quad\left(x_{1}^{2} x_{2} x_{1} x_{2}\right)^{y}=x_{2} .
$$

Let

$$
\begin{gathered}
A=\left\langle x_{1}^{2} x_{2} x_{1} x_{2}, x_{1}\right\rangle=\left\langle x_{2} x_{1} x_{2}, x_{1}\right\rangle, \\
B=\left\langle x_{2}^{-1} x_{1}^{-1} x_{2}^{-1} x_{1}^{-1} x_{2}, x_{2}\right\rangle=\left\langle x_{1} x_{2} x_{1}, x_{2}\right\rangle .
\end{gathered}
$$

Then the group $G_{3}$ is the $H N N$-extension with the stable letter $y$. Groups $A$ and $B$ are 2generated free subgroups in the free group $\left\langle x_{1}, x_{2}\right\rangle$ of rank 2 and the isomorphism $\varphi: A \rightarrow B$ is defined by the rule

$$
x_{1} \mapsto x_{2}^{-1} x_{1}^{-1} x_{2}^{-1} x_{1}^{-1} x_{2}, \quad x_{1}^{2} x_{2} x_{1} x_{2} \mapsto x_{2}
$$

A natural question whether groups $G_{1, r}, G_{2}$ and $G_{3}$ are isomorphic to each other occurs. The next section is dedicated to the answer on this question.

\section{Isomorphism problem}

In the present section the fact that some of virtual trefoil groups listed above are not isomorphic to each other will be proven. For that purpose different approaches will be used: a quotient by the second commutator subgroup, a quotient by terms of lower central series. 


\subsection{Quotient group by the second commutator subgroup}

1) Find the quotient group $G_{2} / G_{2}^{\prime \prime}$. In order to do that note the relation of the group $G_{2}$ :

$$
x y^{-1} x^{-1} y x=y^{-2} x y x^{-1} y^{-1} x y^{2}
$$

is equivalent to the following relation

$$
[x, y]^{2}[x, y]^{2 y}=1 .
$$

Therefore, the quotient group $G_{2} / G_{2}^{\prime \prime}$ can be considered as a cyclic module $G_{2}^{\prime} / G_{2}^{\prime \prime}=\left\langle[x, y] G_{2}^{\prime \prime}\right\rangle$ over the Laurent ring $\mathbb{Z}\left[x^{ \pm 1}, y^{ \pm 1}\right]$. In this module relation can be written in the form

$$
[x, y] \cdot 2(1+y)=0 .
$$

2) Find the quotient group $G_{1, r} / G_{1, r}^{\prime \prime}$. The following commutator identities will be used

$$
[a b, c]=[a, c]^{b}[b, c], \quad[a, b c]=[a, c][a, b]^{c}, \quad\left[a, b^{-1}\right]=[b, a]^{b^{-1}}, \quad[a, b]^{-1}=[b, a] .
$$

Using these identities, the relation of the group $G_{1, r}$ is transformed as follows:

$$
\begin{gathered}
x^{-r} y^{-1} x y x^{r}=y^{-2} x^{r} y x y^{-1} x^{-r} y^{2} \Leftrightarrow \\
\Leftrightarrow x^{-1}\left(y x^{r}\right)^{-1} x\left(y x^{r}\right)=x^{-1}\left(y^{-1} x^{-r} y^{2}\right) x\left(y^{-1} x^{-r} y^{2}\right) \Leftrightarrow \\
\Leftrightarrow\left[x, y x^{r}\right]=\left[x, y^{-1} x^{-r} y^{2}\right] \Leftrightarrow \\
\Leftrightarrow[x, y]^{x^{r}}=\left[x, y^{2}\right]\left[x, y^{-1} x^{-r}\right]^{y^{2}} .
\end{gathered}
$$

Transform the right part of the last relation:

$$
\begin{gathered}
{\left[x, y^{2}\right]\left[x, y^{-1} x^{-r}\right]^{y^{2}}=[x, y][x, y]^{y}\left[x, y^{-1}\right]^{x^{-r} y^{2}}=} \\
=[x, y][x, y]^{y}[y, x]^{y^{-1} x^{-r} y^{2}}=[x, y][x, y]^{y}[x, y]^{-y^{-1} x^{-r} y^{2}} .
\end{gathered}
$$

Thus the relation of the group $G_{1, r}$ by modulo of the second commutator subgroup $G_{1, r}^{\prime \prime}$ has the form

$$
[x, y] \cdot\left(1+y-y x^{-r}-x^{r}\right)=0
$$

or

$$
[x, y] \cdot\left(1-x^{-r}\right)\left(y-x^{r}\right)=0 .
$$

To prove that the group $G_{2}$ is not isomorphic to any group $G_{1, r}$ the following lemma is required. 
Lemma 4.1 If the automorphism of the free metabelian group $F_{2} / F_{2}^{\prime \prime}$ is defined by the action on the generators:

$$
x \mapsto x^{\alpha} y^{\beta}[x, y]^{\gamma}, \quad y \mapsto x^{a} y^{b}[x, y]^{c}, \text { where } \alpha, \beta, \gamma, a, b, c \in \mathbb{Z}, \quad \alpha b-\beta a= \pm 1,
$$

then the commutator $z=[x, y] \in F_{2} / F_{2}^{\prime \prime}$ is transformed by the rule

$$
z \mapsto z \cdot\left(c\left(1-x^{\alpha} y^{\beta}\right)-\gamma\left(1-x^{a} y^{b}\right)+y^{\beta} \frac{\left(1-x^{\alpha}\right)}{(1-x)} \frac{\left(1-y^{b}\right)}{(1-y)}-y^{b} \frac{\left(1-x^{a}\right)}{(1-x)} \frac{\left(1-y^{\beta}\right)}{(1-y)}\right) .
$$

Proof. All further computations are performed in the quotient group $F_{2} / F_{2}^{\prime \prime}$. We have

$$
\begin{gathered}
z \mapsto\left[x^{\alpha} y^{\beta} z^{\gamma}, x^{a} y^{b} z^{c}\right]=\left[x^{\alpha} y^{\beta} z^{\gamma}, z^{c}\right]\left[x^{\alpha} y^{\beta} z^{\gamma}, x^{a} y^{b}\right]= \\
=\left[x^{\alpha} y^{\beta}, z^{c}\right]\left[x^{\alpha} y^{\beta} z^{\gamma}, y^{b}\right]\left[x^{\alpha} y^{\beta} z^{\gamma}, x^{a}\right]^{y^{b}}= \\
=\left[x^{\alpha}, z^{c}\right]^{y^{\beta}}\left[y^{\beta}, z^{c}\right]\left[x^{\alpha} y^{\beta}, y^{b}\right]\left[z^{\gamma}, y^{b}\right]\left[y^{\beta} z^{\gamma}, x^{a}\right] y^{b}= \\
=\left[x^{\alpha}, z^{c}\right]^{y^{\beta}}\left[y^{\beta}, z^{c}\right]\left[x^{\alpha}, y^{b}\right]^{y^{\beta}}\left[z^{\gamma}, y^{b}\right]\left[y^{\beta}, x^{a}\right]^{y^{b}}\left[z^{\gamma}, x^{a}\right] y^{b} .
\end{gathered}
$$

To transform this expression the following equations are used

$$
\left[x^{p}, y^{q}\right]=z \cdot\left(\frac{\left(1-x^{p}\right)}{(1-x)} \frac{\left(1-y^{q}\right)}{(1-y)}\right), \quad\left[x^{p}, z^{q}\right]=z \cdot q\left(1-x^{p}\right),
$$

which are true for arbitrary integers $p, q$. We obtain

$$
\begin{gathered}
z \mapsto z \cdot c\left(1-x^{\alpha}\right) y^{\beta}+z \cdot c\left(1-y^{\beta}\right)+z \cdot y^{\beta} \frac{\left(1-x^{\alpha}\right)}{(1-x)} \frac{\left(1-y^{b}\right)}{(1-y)}+ \\
+z \cdot \gamma\left(y^{b}-1\right)+z \cdot \gamma\left(x^{a}-1\right) y^{b}-z \cdot y^{b} \frac{\left(1-x^{a}\right)}{(1-x)} \frac{\left(1-y^{\beta}\right)}{(1-y)}= \\
=z \cdot\left(c\left(1-x^{\alpha} y^{\beta}\right)-\gamma\left(1-x^{a} y^{b}\right)+y^{\beta} \frac{\left(1-x^{\alpha}\right)}{(1-x)} \frac{\left(1-y^{b}\right)}{(1-y)}-y^{b} \frac{\left(1-x^{a}\right)}{(1-x)} \frac{\left(1-y^{\beta}\right)}{(1-y)}\right) .
\end{gathered}
$$

Using this lemma, the following proposition is proved.

Proposition 4.2 The group $G_{2}$ is not isomorphic to any group $G_{1, r}$.

Proof. Using the transformation

$$
x \mapsto x^{\alpha} y^{\beta} z^{\gamma}, \quad y \mapsto x^{a} y^{b} z^{c}
$$

to the expression $z \cdot 2(1+y)$, we obtain

$$
z \cdot 2\left(1+x^{a} y^{b}\right)\left(c\left(1-x^{\alpha} y^{\beta}\right)-\gamma\left(1-x^{a} y^{b}\right)+y^{\beta} \frac{\left(1-x^{\alpha}\right)}{(1-x)} \frac{\left(1-y^{b}\right)}{(1-y)}-y^{b} \frac{\left(1-x^{a}\right)}{(1-x)} \frac{\left(1-y^{\beta}\right)}{(1-y)}\right) .
$$


If $G_{1, r}$ is isomorphic to the group $G_{2}$ the following equation should be true

$$
\begin{gathered}
2\left(1+x^{a} y^{b}\right)\left(c\left(1-x^{\alpha} y^{\beta}\right)-\gamma\left(1-x^{a} y^{b}\right)+y^{\beta} \frac{\left(1-x^{\alpha}\right)}{(1-x)} \frac{\left(1-y^{b}\right)}{(1-y)}-y^{b} \frac{\left(1-x^{a}\right)}{(1-x)} \frac{\left(1-y^{\beta}\right)}{(1-y)}\right)= \\
=\left(1-x^{-r}\right)\left(y-x^{r}\right)
\end{gathered}
$$

for some parameters $\alpha, \beta, \gamma, a, b, c \in \mathbb{Z}$ and $\alpha b-\beta a= \pm 1$. Setting $x=y=1$ and taking into account that $\left(1-x^{p}\right) /(1-x)=p$ for $x=1$ and for arbitrary integer $p$, we obtain the contradictory equation $\pm 4=0$.

\subsection{Lower central series}

As it is well known for the classical knot group its commutator subgroup coincides with the third term of low central series. Let us show that it is not the case for virtual knots. For example for the group $G_{2}$ it is true

Proposition 4.3 1) The quotient group $\gamma_{2} G_{2} / \gamma_{3} G_{2}$ is isomorphic to the cyclic group of order 4.

2) The quotient group $\gamma_{k} G_{2} / \gamma_{k+1} G_{2}$ is a finite group of exponent 4 for an arbitrary integer $k \geq 2$.

3) The relation of the group $G_{2}$ by modulo $\gamma_{4} G_{2}$ has the form

$$
[y, x]^{4}=[x, y, y]^{2} .
$$

Proof. 1) Transform the relation of the group $G_{2}$

$$
x y^{-1} x^{-1} y x=y^{-2} x y x^{-1} y^{-1} x y^{2} \Rightarrow x[y, x]=y^{-2} x\left[y^{-1}, x\right] y^{2} \Rightarrow x[y, x]=x y^{-2}\left[y^{-2}, x\right]\left[y^{-1}, x\right] y^{2} \text {. }
$$

Therefore, the relation is transformed to the form

$$
[y, x]=y^{-2}\left[y^{-2}, x\right]\left[y^{-1}, x\right] y^{2}
$$

By modulo $\gamma_{3} G_{2}$ we have

$$
[y, x]^{4}=1 .
$$

2) Since the quotient group $\gamma_{k} G_{2} / \gamma_{k+1} G_{2}$ is generated by left-normalized commutators of the form

$$
\left[\ldots\left[g_{1}, g_{2}\right], \ldots, g_{k}\right], \quad g_{i} \in G_{2},
$$

raising to the forth power and using relations of the quotient group $\gamma_{k} G_{2} / \gamma_{k+1} G_{2}$, we obtain

$$
\left[\ldots\left[g_{1}, g_{2}\right], \ldots, g_{k}\right]^{4}=\left[\ldots\left[g_{1}, g_{2}\right]^{4}, \ldots, g_{k}\right]=1
$$

due to the proved item 1). 
3) Transform the right part of the relation

$$
[y, x]=y^{-2}\left[y^{-2}, x\right]\left[y^{-1}, x\right] y^{2}
$$

by modulo $\gamma_{4} G_{2}$. We have

$$
\begin{gathered}
y^{-2}\left[y^{-2}, x\right]\left[y^{-1}, x\right] y^{2}=\left[y^{-2}, x^{y^{2}}\right]\left[y^{-1}, x^{y^{2}}\right]=\left[y^{-2}, x\left[x, y^{2}\right]\right]\left[y^{-1}, x\left[x, y^{2}\right]\right]= \\
=\left[y^{-2},\left[x, y^{2}\right]\right]\left[y^{-2}, x\right]\left[y^{-1},\left[x, y^{2}\right]\right]\left[y^{-1}, x\right]=[y,[x, y]]^{-4}\left[y^{-2}, x\right][y,[x, y]]^{-2}\left[y^{-1}, x\right]= \\
=\left[y^{-2}, x\right]\left[y^{-1}, x\right][x, y, y]^{6}=\left[y^{-1}, x\right]^{y^{-1}}\left[y^{-1}, x\right]^{2}[x, y, y]^{6}= \\
=\left[y^{-1}, x\right]\left[y^{-1}, x, y^{-1}\right]\left[y^{-1}, x\right]^{2}[x, y, y]^{6}=\left[y^{-1}, x\right]^{3}[x, y, y]^{5}= \\
=\left([x, y]^{y^{-1}}\right)^{3}[x, y, y]^{5}=\left([x, y]\left[x, y, y^{-1}\right]\right)^{3}[x, y, y]^{5}=[x, y]^{3}[x, y, y]^{2} .
\end{gathered}
$$

Thus the relation of the group $G_{2}$ by modulo $\gamma_{4} G_{2}$ has the form

$$
[y, x]=[x, y]^{3}[x, y, y]^{2},
$$

which is equivalent to

$$
[y, x]^{4}=[x, y, y]^{2}
$$

Remark. The relation

$$
[y, x]^{4}=[x, y, y]^{2}
$$

of the group $G_{2}$ by modulo $\gamma_{4} G_{2}$ can be presented in the form

$$
\left([y, x]^{2}\right)^{y}=[y, x]^{-2} .
$$

Indeed

$$
[y, x]^{4}=[x, y, y]^{2}=\left[[y, x]^{-2}, y\right]=[y, x]^{2} y^{-1}[y, x]^{-2} y
$$

Consider terms of the lower central series of the group $G_{1, r}$. It is true

Theorem 4.4 The first five terms of low central series of the group $G_{1, r}$ are different from each other. Moreover $G_{1, r} / \gamma_{4} G_{1, r} \cong F_{2} / \gamma_{4} F_{2}, \gamma_{4} G_{1, r} / \gamma_{5} G_{1, r} \cong \mathbb{Z} \times \mathbb{Z} \times \mathbb{Z}_{r}$.

Proof. At first let us show that the relation of the group $G_{1, r}$ by modulo of the subgroup $\gamma_{5} G_{1, r}$ has the form

$$
[y, x, y, x]^{r}=[y, x, x, y]^{r^{2}} .
$$

For that purpose rewrite the relation of the group $G_{1, r}$ in the form

$$
x^{y x^{r}}=x^{y^{-1} x^{-r} y^{2}} .
$$


It is equivalent to

$$
\left[x, y x^{r} y^{-2} x^{r} y\right]=1 .
$$

Using commutator identities, transform the left side of this relation:

$$
\begin{gathered}
{\left[x, y x^{r} y^{-2} x^{r} y\right]=\left[x,\left(y x^{r} y^{-1}\right)\left(y^{-1} x^{r} y\right)\right]=\left[x,\left[y^{-1}, x^{-r}\right] x^{2 r}\left[x^{r}, y\right]\right]=} \\
=\left[x,\left[x^{r}, y\right]\right]\left[x,\left[y^{-1}, x^{-r}\right] x^{2 r}\right]^{\left[x^{r}, y\right]}=\left[x,\left[x^{r}, y\right]\right]\left[x,\left[y^{-1}, x^{-r}\right]\right]^{x^{2 r}\left[x^{r}, y\right]}= \\
=\left[x,\left[x^{r}, y\right]\right]\left[x,\left[y, x^{r}\right]^{y^{-1} x^{-r}}\right]^{x^{2 r}\left[x^{r}, y\right]}=\left[x,\left[x^{r}, y\right]\right]\left[x,\left[y, x^{r}\right]\left[y, x^{r}, y^{-1} x^{-r}\right]\right]^{x^{2 r}\left[x^{r}, y\right]}= \\
\left.=\left[x,\left[x^{r}, y\right]\right]\left[x,\left[y, x^{r}, y^{-1} x^{-r}\right]\right]^{x^{2 r}\left[x^{r}, y\right]}\left[x,\left[y, x^{r}\right]\right]\right]^{\left[y, x^{r}, y^{-1} x^{-r}\right] x^{2 r}\left[x^{r}, y\right]}= \\
=\left[x,\left[x^{r}, y\right]\right]\left[x,\left[y, x^{r}, y^{-1} x^{-r}\right]\right]\left[\left[x,\left[y, x^{r}, y^{-1} x^{-r}\right]\right], x^{2 r}\left[x^{r}, y\right]\right] \times \\
\times\left[x,\left[y, x^{r}\right]\right]\left[\left[x,\left[y, x^{r}\right]\right],\left[y, x^{r}, y^{-1} x^{-r}\right] x^{2 r}\left[x^{r}, y\right]\right]=
\end{gathered}
$$

(by modulo $\gamma_{5} G_{1, r}$ we obtain)

$$
\begin{gathered}
=\left[x,\left[x^{r}, y\right]\right]\left[x,\left[y, x^{r}, y^{-1} x^{-r}\right]\right]\left[x,\left[y, x^{r}\right]\right]\left[\left[x,\left[y, x^{r}\right]\right], x^{2 r}\right]= \\
=\left[x,\left[x^{r}, y\right]\right]\left[x,\left[y, x^{r}\right]\right][x,[y, x, y]]^{-r}[x,[y, x, x]]^{-r^{2}}[y, x, x, x]^{-2 r^{2}}= \\
=\left[x,\left[x^{r}, y\right]\right]\left[x,\left[x^{r}, y\right]^{-1}\right][y, x, y, x]^{r}[y, x, x, x]^{-r^{2}}= \\
=\left[x,\left[x^{r}, y\right]\right]\left[\left[x^{r}, y\right], x\right]^{\left[x^{r}, y\right]^{-1}}[y, x, y, x]^{r}[y, x, x, x]^{-r^{2}}= \\
=[y, x, y, x]^{r}[y, x, x, x]^{-r^{2}} .
\end{gathered}
$$

Recall that $[y, x, y, x] \equiv[y, x, x, y]\left(\bmod \gamma_{5} F_{2}\right)$. Indeed for $a=[y, x], b=y, c=x$ from Hall identity for $F_{3} / \gamma_{4} F_{3}$ we have

$$
[a, b, c][b, c, a][c, a, b]=1 \text {. }
$$

Due to $a=[b, c]$ we obtain

$$
[y, x, y, x][x,[y, x], x] \equiv 1\left(\bmod \gamma_{5} G_{1, r}\right)
$$

or

$$
[y, x, y, x] \equiv[y, x, x, y]\left(\bmod \gamma_{5} F_{2}\right) .
$$

Therefore, the relation of the group $G_{1, r}$ by modulo of the subgroup $\gamma_{5} G_{1, r}$ has the form

$$
[y, x, y, x]^{r}=[y, x, x, x]^{r^{2}} \text {. }
$$

Basic commutators of weight 4 are of the form $c_{1}=[y, x, x, x], c_{2}=[y, x, x, y], c_{3}=$ $[y, x, y, y]$. Hence the relation of the quotient group $\gamma_{4} G_{1, r} / \gamma_{5} G_{1, r}$ can be written in the form $\left(c_{2} c_{1}^{-r}\right)^{r}=1$. 


\section{The proof of non-triviality of Kishino knot}

It was proved in [13] that groups $G_{1, r}(K i)$ and $G_{2}(K i)$ cannot distinguish the Kishino knot $K i$ from the trivial one. Also in [13] the question was placed whether the group $G_{3}(K i)$ is able to distinguish the Kishino knot from the trivial one or not. Note that the group $G_{3}(U)$ of the trivial knot $U$ is isomorphic to $F_{2}$. In the present section the positive answer on the question will be given. For that purpose the fact that the group $G_{3}(K i)$ is not isomorphic to the free group $F_{2}$ is proved. To do that the Magnus representation of a free metabelian group by matrices $2 \times 2$ is required. Recall the Magnus representation (see [12, Chapter 1, Section 4]).

Let $R$ be a commutative ring over $\mathbb{Z}$ containing independent invertible elements $s_{1}, \ldots, s_{q}$ and $t_{1}, \ldots, t_{q}$. Magnus showed that the map $\varphi$ from the free metabelian group $F_{q} / F_{q}^{\prime \prime}$ into $\mathrm{GL}_{2}(R)$ defined by

$$
x_{i} \varphi=\left(\begin{array}{cc}
s_{i} & t_{i} \\
0 & 1
\end{array}\right), \quad i=1, \ldots, q,
$$

is a monomorphism.

Using the Magnus representation, the following theorem is stated.

Theorem 5.1 The group $G=G_{3}(K i)$ having generators $a, b, c, d$ and the system of defining relations

$$
\begin{gathered}
d^{-1} l^{-d} c^{-2 d^{-1}} b^{-d} c^{-2 d^{-1}} a a^{-2 d} d=a^{-1} b^{-d} c^{-2 d^{-1}} a, \\
c^{-1} b c=b^{-d} c^{d^{-1}} b^{d}, \\
c=b^{-d} c^{-2 d^{-1}} b^{-d} c^{-2 d^{-1}} a a^{-d} a^{-1} c^{2 d^{-1}} b^{2 d} .
\end{gathered}
$$

is not isomorphic to the free group of rank 2.

Proof. Proof by contradiction. Assume that $G$ is generated freely by some elements $x, y$, i. e. $G=\langle x, y\rangle \cong F_{2}$. Consider the Magnus representation $\varphi: G \longrightarrow \mathrm{GL}_{2}(R)$ defined by the action on generators:

$$
x \mapsto\left(\begin{array}{cc}
s_{1} & t_{1} \\
0 & 1
\end{array}\right), \quad y \mapsto\left(\begin{array}{cc}
s_{2} & t_{2} \\
0 & 1
\end{array}\right) .
$$

Here $s_{1}$ and $s_{2}$ are free generators of a free abelian group of rank 2 . Consider elements $t_{1}, t_{2}$ as generators of a free module $M$ over the Laurent ring $\mathbb{Z}\left[s_{1}^{ \pm 1}, s_{2}^{ \pm 1}\right]$.

The representation maps the generators $a, b, c, d$ of the group $G$ to matrices

$$
a \mapsto\left(\begin{array}{cc}
A & \gamma \\
0 & 1
\end{array}\right), \quad b \mapsto\left(\begin{array}{cc}
B & \lambda \\
0 & 1
\end{array}\right), \quad c \mapsto\left(\begin{array}{cc}
C & \mu \\
0 & 1
\end{array}\right), \quad d \mapsto\left(\begin{array}{cc}
D & \nu \\
0 & 1
\end{array}\right),
$$

where $A, B, C, D \in\left\langle s_{1}, s_{2}\right\rangle, \gamma, \lambda, \mu, \nu \in M$. 
The projection of $\varphi(G)$ to the entry $(1,1)$ defines the homomorphism of the group $G$ onto the group $\langle A, B, C, D\rangle \leq\left\langle s_{1}, s_{2}\right\rangle$ with the kernel $G^{\prime}$. Using relations (1)-(3) in the quotient group $G / G^{\prime}$, the following relations are obtained:

$$
A B C^{2}=1, \quad B=C, \quad A C^{3}=1 .
$$

Thus

$$
A=C^{-3}, \quad B=C .
$$

Therefore, the representation $\varphi$ acts on the generators of the group $G$ in the following manner:

$$
a \mapsto\left(\begin{array}{cc}
C^{-3} & \gamma \\
0 & 1
\end{array}\right), \quad b \mapsto\left(\begin{array}{cc}
C & \lambda \\
0 & 1
\end{array}\right), \quad c \mapsto\left(\begin{array}{cc}
C & \mu \\
0 & 1
\end{array}\right), \quad d \mapsto\left(\begin{array}{cc}
D & \nu \\
0 & 1
\end{array}\right) .
$$

Is it clear to see that $\langle C, D\rangle=\left\langle s_{1}, s_{2}\right\rangle$.

As a further step consider the relation (2). Using the homomorphism $\varphi$, the equality is obtained

$$
\begin{gathered}
\left(\begin{array}{cc}
C & C^{-1} \lambda+\left(1-C^{-1}\right) \mu \\
0 & 1
\end{array}\right)= \\
=\left(\begin{array}{cc}
C & D^{-1}\left(1-C^{-1}\right) \lambda+C^{-1} D \mu+\left(D^{-1}(C-1)+\left(C^{-1}-1\right)\left(D^{-1}+1\right)\right) \nu \\
0 & 1
\end{array}\right) .
\end{gathered}
$$

Hence, in the module $M$ there is the relation

$$
C^{-1} \lambda+\left(1-C^{-1}\right) \mu=D^{-1}\left(1-C^{-1}\right) \lambda+C^{-1} D \mu+\left(D^{-1}(C-1)+\left(C^{-1}-1\right)\left(D^{-1}+1\right)\right) \nu
$$

or

$$
\left(C^{-1}+D^{-1}\left(C^{-1}-1\right)\right) \lambda+\left(1-C^{-1}-C^{-1} D\right) \mu+\left(1-C^{-1}\right)\left(D^{-1}+1-D^{-1} C\right) \nu=0 .
$$

Multiplying by $C D$, we obtain

$$
(D+1-C) \lambda+D(C-1-D) \mu+(C-1)(1+D-C) \nu=0,
$$

which is equivalent to the relation

$$
(1-C+D)(\lambda-D \mu+(C-1) \nu)=0 .
$$

Since the module $M$ is torsion free

$$
\lambda-D \mu+(C-1) \nu=0 .
$$

Rewrite this relation in the form

$$
D \mu+\nu=\lambda+C \nu .
$$


Acting by the homomorphism $\varphi$ on the products $d c$ and $b d$, we obtain

$$
\varphi(d c)=\left(\begin{array}{cc}
C D & D \mu+\nu \\
0 & 1
\end{array}\right), \quad \varphi(b d)=\left(\begin{array}{cc}
C D & \lambda+C \nu \\
0 & 1
\end{array}\right) .
$$

Due to (4) we have the equality $\varphi(d c)=\varphi(b d)$, i. e. $\varphi\left(c^{-1} b^{d}\right)=1$. Since the Magnus representation is a faithful representation of the free metabelian group, the following relation ought to hold in the quotient group $G / G^{\prime \prime}$

$$
c=b^{d} \text {. }
$$

Let us prove the following lemma.

Lemma 5.2 The quotient group of the group $G$ by the normal closure of $c^{-1} b^{d}$ is isomorphic to the group

$$
G_{1}=\left\langle a, c, d \| c^{-1} c^{-2 d^{-1}}=a a^{d} a^{-1}\right\rangle .
$$

Proof. After the substitution $b^{d}=c$ the relation (2) becomes

$$
c^{-1} b c=c^{-1} c^{d^{-1}} c \Rightarrow b=c^{d^{-1}} \Leftrightarrow c=b^{d},
$$

i. e. it is identity due to (5).

After the substitution $b^{d}=c$ the relation (3) is of the form

$$
\begin{gathered}
c=c^{-1} c^{-2 d^{-1}} c^{-1} c^{-2 d^{-1}} a a^{-d} a^{-1} c^{2 d^{-1}} c^{2} \Rightarrow \\
1=c^{-2 d^{-1}} c^{-1} c^{-2 d^{-1}} a a^{-d} a^{-1} c^{2 d^{-1}} \Leftrightarrow \\
c^{-1} c^{-2 d^{-1}}=a a^{d} a^{-1} .
\end{gathered}
$$

After the substitution $b^{d}=c$ the relation (1) becomes

$$
d^{-1} c^{-1} c^{-2 d^{-1}} c^{-1} c^{-2 d^{-1}} a a^{-2 d} d=a^{-1} c^{-1} c^{-2 d^{-1}} a .
$$

Substituting the product $c^{-1} c^{-2 d^{-1}}=a a^{d} a^{-1}$ due to (6), we obtain

$$
\begin{gathered}
d^{-1} a a^{d} a^{-1} a a^{d} a^{-1} a a^{-2 d} d=a^{-1} a a^{d} a^{-1} a \Rightarrow \\
d^{-1} a a^{d} a^{d} a^{-2 d} d=a^{d} \Rightarrow \\
d^{-1} a d=a^{d}
\end{gathered}
$$

- the identity.

Thus the quotient group has only one defining relation (6).

Denote by $F_{3}=\langle a, c, d\rangle$ a free group of rank 3 with free generators $a, c, d$. Due to the assumption on the group $G$ and in view of the lemma 5.2 the group

$$
\left\langle a, c, d \| c c^{2 d^{-1}} a a^{d} a^{-1}=1, F_{3}^{\prime \prime}\right\rangle
$$


is isomorphic to the free metabelian group $F_{2} / F_{2}^{\prime \prime}$ of rank 2 .

Due to results of the paper [10] it is possible if and only if the element

$$
w=c c^{2 d^{-1}} a a^{d} a^{-1} \in F_{3}
$$

is a primitive in the free metabelian group $F_{3} / F_{3}^{\prime \prime}$. Due to [15] the element $w$ is primitive in $F_{3} / F_{3}^{\prime \prime}$ if and only if the vector

$$
\left(\partial_{a} w, \partial_{c} w, \partial_{d} w\right)
$$

is unimodular (i. e. the ideal generated by its components contains 1 in the Laurent ring $\left.\mathbb{Z}\left[a^{ \pm 1}, c^{ \pm 1}, d^{ \pm 1}\right]\right)$. Here $\partial_{a} w, \partial_{c} w, \partial_{d} w$ are left Fox derivatives by variables $a, c, d$ respectively (see [12, Chapter 1, Section 10]).

Values of Fox derivatives are taken in the Laurent ring $\mathbb{Z}\left[F_{3} / F_{3}^{\prime}\right]$. Hence, to simplify the notation the assumption $F_{3} / F_{3}^{\prime}=\langle a, c, d\rangle$ is made, i. e. $a, c, d$ are generators of the free abelian group. We have

$$
\begin{gathered}
\partial_{a} w=c^{3}\left(1+d^{-1} a-a\right), \\
\partial_{c} w=1+c d+c^{2} d, \\
\partial_{d} w=c-c^{3}-c^{3} a d^{-1}+c^{3} a^{2} d^{-1} .
\end{gathered}
$$

Pass from the vector $\left(\partial_{a} w, \partial_{c} w, \partial_{d} w\right)$ to the vector

$$
\begin{gathered}
\left(c^{-3} d \partial_{a} w, \partial_{c} w, c^{-1} d \partial_{d} w\right)= \\
=\left(a+d-a d, 1+c d+c^{2} d, d-c^{2} d-c^{2} a+c^{2} a^{2}\right) .
\end{gathered}
$$

Due to assumption on the unimodularity there are coefficients

$$
A, C, D \in \mathbb{Z}\left[a^{ \pm 1}, c^{ \pm 1}, d^{ \pm 1}\right]
$$

such that the following equation holds

$$
(a+d-a d) A+\left(1+c d+c^{2} d\right) C+\left(d-c^{2} d-c^{2} a+c^{2} a^{2}\right) D=1 .
$$

Multiplying by a suitable monomial of the form $a^{\gamma} c^{\pi} d^{\nu}$, where $\gamma, \pi, \nu$ are positive integers, we obtain

$$
(a+d-a d) A^{\prime}+\left(1+c d+c^{2} d\right) C^{\prime}+\left(d-c^{2} d-c^{2} a+c^{2} a^{2}\right) D^{\prime}=a^{\gamma} c^{\pi} d^{\nu}
$$

which holds in the polynomial ring, $A^{\prime}, C^{\prime}, D^{\prime} \in \mathbb{Z}[a, c, d]$.

Consider the system of equations

$$
\begin{gathered}
a+d-a d=0, \\
1+c d+c^{2} d=0, \\
d-c^{2} d-c^{2} a+c^{2} a^{2}=0 .
\end{gathered}
$$


It follows from (9) that

$$
d=-\frac{1}{c^{2}+c} .
$$

From (8) due to (11) we have

$$
a=\frac{1}{c^{2}+c+1} .
$$

From (10) due to (11) and (12) the equation on $c$ is obtained

$$
c^{3}-c^{2}-c-1=0 .
$$

Let $c_{0}$ be some solution of (13), generally speaking a complex number. Since system of equations

$$
\left\{\begin{array} { l } 
{ c ^ { 2 } + c = 0 , } \\
{ c ^ { 3 } - c ^ { 2 } - c - 1 = 0 }
\end{array} \quad \text { and } \quad \left\{\begin{array}{l}
c^{2}+c+1=0, \\
c^{3}-c^{2}-c-1=0
\end{array}\right.\right.
$$

are not compatible,

$$
d_{0}=-\frac{1}{c_{0}^{2}+c_{0}}, \quad a_{0}=\frac{1}{c_{0}^{2}+c_{0}+1}
$$

are well defined. Note also that $a_{0}, c_{0}, d_{0} \neq 0$. Substituting $a=a_{0}, c=c_{0}, d=d_{0}$ in (7), we obtain

$$
0=a_{0}^{\gamma} c_{0}^{\pi} d_{0}^{\nu} \neq 0
$$

which is a contradictory expression. Thus the vector $\left(\partial_{a} w, \partial_{c} w, \partial_{d} w\right)$ is not unimodular. Therefore, the group $G$ is not a free group.

\section{Representations of groups by formal power series}

In the present section we will show that for the studying of virtual knot groups we can use representations by rings of formal power series with non-commutative variables.

Recall (see, for example [12, Chapter 1, Proposition 10.1]) a well known representation of a free group $F_{n}=\left\langle x_{1}, x_{2}, \ldots, x_{n}\right\rangle$ into the ring of formal power series $\mathbb{Z}\left[\left[X_{1}, X_{2}, \ldots, X_{n}\right]\right]$ of noncommutative variables $X_{1}, X_{2}, \ldots, X_{n}$ defined by the action on generators:

$$
x_{i} \mapsto 1+X_{i}, \quad i=1,2, \ldots, n .
$$

In that case inverse elements of generators go to the following formal power series

$$
x_{i}^{-1} \mapsto 1-X_{i}+X_{i}^{2}-X_{i}^{3}+\ldots, \quad i=1,2, \ldots, n .
$$


The representation defined in that manner is faithful (i. e. its kernel is trivial). Moreover, it remains being faithful if the ring $\mathbb{Z}\left[\left[X_{1}, X_{2}, \ldots, X_{n}\right]\right]$ is replaced by the quotient ring $\mathbb{Z}\left[\left[X_{1}, X_{2}, \ldots, X_{n}\right]\right] /\left\langle X_{1}^{2}, X_{2}^{2}, \ldots, X_{n}^{2}\right\rangle$ by the two-sided ideal generated by elements $X_{1}^{2}, X_{2}^{2}, \ldots, X_{n}^{2}$.

Let

$$
\mathcal{P}=\left\langle x_{1}, \ldots, x_{n} \| r_{1}, \ldots, r_{m}\right\rangle
$$

be some finite presentation of the group $G$ and $A_{n}=\mathbb{Q}\left[\left[X_{1}, \ldots, X_{n}\right]\right]$ is an algebra of formal power series of noncommutative variables $X_{1}, \ldots, X_{n}$ over the field of rational numbers. Define series $f_{j}, j=1, \ldots, m$, in the algebra $A_{n}$ by equalities

$$
f_{j}=r_{j}\left(1+X_{1}, \ldots, 1+X_{n}\right)-1, \quad j=1, \ldots, m .
$$

Proposition 6.1 The quotient algebra $A_{n} /\left\langle f_{1}, \ldots, f_{m}\right\rangle$ is the invariant of the group $G$, $i$. e. it does not depend on the explicit presentation.

Proof. It is sufficient to show that the quotient algebra $A_{n} /\left\langle f_{1}, \ldots, f_{m}\right\rangle$ is unaltered in case the presentation $\mathcal{P}$ is changed by Tietze transformations.

1. In case of bringing in a new generator $z=w\left(x_{1}, \ldots, x_{n}\right)$ a new variable $Z$ and the relation of the form $Z=W\left(X_{1}, \ldots, X_{n}\right)$ are added to the algebra

$$
A_{n} /\left\langle f_{1}, \ldots, f_{m}\right\rangle
$$

where $W\left(X_{1}, \ldots, X_{n}\right)=w\left(1+X_{1}, \ldots, 1+X_{n}\right)-1$. Hence the quotient algebra $A_{n} /\left\langle f_{1}, \ldots, f_{m}\right\rangle$ is unaltered. Analogously in case of excluding a generator the quotient algebra $A_{n} /\left\langle f_{1}, \ldots, f_{m}\right\rangle$ stays unaltered.

2. In case of bringing in the relation of the form $r_{p} r_{q}$ in the group $G$ the relation $f_{p}+f_{q}-f_{p} f_{q}$ is added to the algebra, which does not change the ideal $\left\langle f_{1}, \ldots, f_{m}\right\rangle$. By the analogy in case of excluding the relation the quotient algebra $A_{n} /\left\langle f_{1}, \ldots, f_{m}\right\rangle$ is unaltered.

Considering quotients of $A_{n}$ by some ideals, we can construct representations of groups in finite dimensional algebras. For example, consider the quotient $B_{2}=\mathbb{Q}[[X, Y]] /\left\langle X^{2}, Y^{2}\right\rangle$ and prove

Proposition 6.2 An arbitrary nontrivial quotient algebra of the algebra $B_{2}$ has a finite dimension.

Proof. Let $f=f(X, Y)$ be a nontrivial polynomial in the algebra $B_{2}$. Then its summand of the highest power has one of the following form

$$
a(X Y)^{k}+b(Y X)^{k}, \quad a(X Y)^{k} X+b(Y X)^{k} Y, \quad a, b \in \mathbb{Q} .
$$

Assume at first that

$$
f=g+a(X Y)^{k}+b(Y X)^{k}
$$


where the power of element $g \in B_{2}$ is strictly less than $2 k$. Suppose that $a \neq 0$. Multiplying this equation by $X$ on the right and by $Y$ on the left, we obtain

$$
f X=g X+a(X Y)^{k} X, \quad Y f=Y g+a Y(X Y)^{k} .
$$

Therefore, in the quotient algebra $B_{2} /\langle f\rangle$ monomials of the power at least $2 k+1$ can be expressed through monomials of the power at most $2 k$, i. e. the dimension of the quotient algebra $B_{2} /\langle f\rangle$ is at most $4 k+1$.

The second case:

$$
f=g+a(X Y)^{k} X+b(Y X)^{k} Y
$$

is considered by analogy.

Let us construct representations of the group $G_{1, r}$ and $G_{2}$ in $B_{2}$. Let the generators of $G_{1, r}$ go to the elements:

$$
x \mapsto 1+X, \quad y \mapsto 1+Y
$$

of $B_{2}$. Then

$$
x^{-1} \mapsto 1-X, \quad y^{-1} \mapsto 1-Y .
$$

Find out the additional relation on variables $X, Y$ in such way that the map

$$
x \mapsto 1+X, \quad y \mapsto 1+Y
$$

can be extended to the representation of the group $G_{1, r}$.

If we go to the algebra than the relation of the group $G_{1, r}$ has the form

$$
\begin{aligned}
(1-r X)(1-Y)(1 & +X)(1+Y)(1+r X)= \\
& =(1-2 Y)(1+r X)(1+Y)(1+X)(1-Y)(1-r X)(1+2 Y) .
\end{aligned}
$$

Opening the brackets and collecting similar terms, the following relation is obtained

$$
(X Y)^{2}-(Y X)^{2} Y+r(X Y)^{3}-r^{2}(Y X)^{3}-2 r^{2}(Y X)^{3} Y=0
$$

This relation should be added to the algebra $B_{2}$ to get the representation of the group $G_{1, r}$. Multiply the last relation by $Y$ on the left

$$
(Y X)^{2} Y+r(Y X)^{3} Y=0
$$

Since $1+r X Y$ is the invertible element, $(Y X)^{2} Y=0$. Multiplying the relation by $X$ on the right, we have

$$
(X Y)^{2} X-(Y X)^{3}+r(X Y)^{3} X-2 r^{2}(Y X)^{4}=0 .
$$

Due to $(Y X)^{2} Y=0$ we obtain $(X Y)^{2} X=0$. Thus the initial relation becomes $(X Y)^{2}=0$.

Therefore, the following proposition is proved. 
Proposition 6.3 The group $G_{1, r}$ allows the representation

$$
x \mapsto 1+X, \quad y \mapsto 1+Y
$$

into the group of invertible elements of the algebra $B_{2} /\left\langle(X Y)^{2}\right\rangle$.

Remark 6.4 Note that the algebra $B_{2} /\left\langle(X Y)^{2}\right\rangle$ is a vector space over the field $\mathbb{Q}$ of dimension 8 with the basis $1, X, Y, X Y, Y X, X Y X, Y X Y,(Y X)^{2}$. Hence, it is possible to construct a linear representation of $G_{1, r}$.

Consider now the group $G_{2}$ and construct a map into the algebra $B_{2}$. Find out the additional relation on variables $X, Y$ in such a manner that the map

$$
x \mapsto 1+X, \quad y \mapsto 1+Y
$$

can be extended to the representation of the group $G_{2}$.

The relation of the group $G_{2}$ passing into the algebra becomes

$$
\begin{aligned}
(1+X)(1-Y)(1-X) & (1+Y)(1+X)= \\
= & (1-2 Y)(1+X)(1+Y)(1-X)(1-Y)(1+X)(1+2 Y) .
\end{aligned}
$$

Opening brackets and collecting similar terms, the following relation is obtained

$$
2 X Y-2 Y X-4 Y X Y-(Y X)^{3}+(X Y)^{3}-2(Y X)^{3} Y=0 .
$$

This relation should be added to the algebra $B_{2}$ to get the representation of the group $G_{2}$.

Multiply the relation by $Y$ on the left

$$
2 Y X Y+(Y X)^{3} Y=0 .
$$

Since $2+(X Y)^{2}$ is the invertible element, $Y X Y=0$. Multiplying the relation by $X$ on the right, we have

$$
2 X Y X-4(Y X)^{2}+(X Y)^{3} X-2(Y X)^{4}=0
$$

Due to the equality $Y X Y=0$ we obtain $X Y X=0$. Hence the initial relation becomes of the form $X Y=Y X$.

Therefore, it has been proven

Proposition 6.5 The group $G_{2}$ allows the representation

$$
x \mapsto 1+X, \quad y \mapsto 1+Y
$$

into the algebra $B_{2} /\langle X Y-Y X\rangle$.

Note that the algebra $\mathcal{A}_{2} /\langle X Y-Y X\rangle$ is a vector space over the field $\mathbb{Q}$ of dimension 4 with the basis $1, X, Y, X Y$. 


\section{Open questions}

In conclusion we formulate series of open questions.

If $K$ is a classical knot than $G_{1,1}(K)$ is a classical knot group, i. e. a fundamental group of the compliment of $K$ in 3-dimensional sphere. Nelson and Neumann proved [14] that the group $G_{1,1}(K)$ is a subgroup of the group $G_{1, r}(K)$ for arbitrary $r \geq 1$ and in contrast to $G_{1,1}(K)$ it determines the knot $K$ up to reflection.

Problem 7.1 1) Is it true for links?

2) Is it possible to prove the similar statement for groups $G_{3}(K)$, which constructed by using the extension of the Wada representation $W_{3}$ ?

Let $G_{2}$ be the virtual trefoil group defined above.

Problem 7.2 1) Does the equation $\gamma_{\omega} G_{2}=\gamma_{\omega+1} G_{2}$ hold?

2) Is there the isomorphism

$$
\gamma_{k} G_{2} / \gamma_{k+1} G_{2} \cong\left(\gamma_{k} F_{2} / \gamma_{k+1} F_{2}\right) \otimes \mathbb{Z}_{4}
$$

for arbitrary integer $k \geq 2$ ?

Problem 7.3 Is there a virtual knot $K$ for which some group from the set $\left\{G_{1,1}(K), G_{2}(K), G_{3}(K)\right\}$ is residually nilpotent?

\section{References}

[1] V. G. Bardakov, Virtual and welded links and their invariants. Sib. Elektron. Mat. Izv., 2 (2005), 196-199 (electronic).

[2] V. G. Bardakov, P. Bellingeri, Groups of virtual and welded links, J. Knot Theory Ramifications, 23, no. 3 (2014), 1450014, 23 pp.

[3] V. G. Bardakov, R. V. Mikhailov, On the residual properties of link groups. (Russian) Sibirsk. Mat. Zh., 48, no. 3 (2007), 485-495; translation in Siberian Math. J. 48, no. 3 (2007), 387-394.

[4] V. G. Bardakov, Yu. A. Mikhalchishina, M. V. Neshchadim, Representations of virtual braids by automorphisms and virtual knot groups, J. Knot Theory Ramifications, 2017, 26, 1750003, 17 pp. 
[5] V. G. Bardakov, Yu. A. Mikhalchishina, M. V. Neshchadim, Virtual link groups, Siberian Mathematical Journal, 58, no. 5 (2017), 765-777.

[6] V. G. Bardakov, M. V. Neshchadim, Knot groups and resudually nilpotence, Procedengs of the Institute of Mathematics and mekhanics Ural brans of RAS, 23, no. 4 (2017), 43-51.

[7] J. S. Birman, Braids, Links, and Mapping Class Groups, Annals of Math. Studies 82, Princeton University Press, 1974.

[8] H. U. Boden, E. Dies, A. I. Gaudreau, A. Gerlings, E. Harper, A. J. Nicas, Alexander invariants for virtual knots. J. Knot Theory Ramifications, 24, no. 3 (2015), 1550009, $62 \mathrm{pp}$.

[9] J. S. Carter, D. Silver, S. Williams, Invariants of links in thickened surfaces, Algebr. Geom. Topol., 14 (2014), no. 3, 1377-1394.

[10] C. K. Gupta, N. D. Gupta, G. A. Noskov, Some applications of Artamonov-QuillenSuslin theorems to metabelian inner rank and primitivity, Canad. J. Math., 46, no. 2 (1994), 298-307.

[11] L. H. Kauffman, Virtual knot theory, Eur. J. Comb., 20, no. 7 (1999), 663-690.

[12] R. C. Lyndon, P. E. Schupp, Combinatorial group theory. Ergebnisse der Mathematik und ihrer Grenzgebiete, Band 89. Springer-Verlag, Berlin-New York, 1977. xiv+339 pp. ISBN: 3-540-07642-5.

[13] Yu. A. Mikhalchishina, Generalizations of Wada representations and virtual link groups. (Russian) Sibirsk. Mat. Zh., 58, no. 3 (2017), 641-659.

University Press, Princeton, NJ, 1957.

[14] S. Nelson, W. Neumann. The 2-generalized knot group determines the knot, Commun. Contemp. Math., 10, suppl. 1 (2008), 843-847.

[15] V. A. Roman'kov, Criteria for the primitivity of a system of elements of a free metabelian group. (Russian) Ukrain. Mat. Zh., 43, no. 7-8 (1991), 996-1002; translation in Ukrainian Math. J. 43 (1991), no. 7-8, 930-935 (1992).

[16] D. Silver, S. G. Williams, Alexander groups and virtual links, J. Knot Theory Ramifications, 10, no. 1 (2001), 151-160.

[17] M. Wada, Group invariants of links, Topology, 31, no. 2 (1992), 399-406. 